\section{Chronic lung disease and occupational dust exposure}

SIR,-We are currently reviewing the evidence concerning the relation of chronic obstructive lung disease or chronic bronchitis and occupational exposure to dust. To avoid bias in the selection of epidemiological studies, we are conducting a comprehensive search for both published and unpublished relevant research and we are using explicitly a priori criteria for the inclusion of studies. We are seeking information on studies that have been completed, but for one reason or another, have not been published. If readers know of such data, it would be appreciated if they would contact us at the address below.

D C F MUIB

S STOCK

Occupational Health Program,

McMaster University, 1200 Main Street West,

Hamilton, Ontario, L8N 3Z5,

Canada. 